\section{IMMUNOLOGY AND INFECTIOUS DISEASES}

\section{G180 NEUTROPHILS OF STRESSED AND HEALTHY NEONATES ARE MORE SENSITIVE TO STIMULATION THAN ADULT NEUTROPHILS}

\section{NMR Wilkinson, AR Bedford Russell and EG Davies St Georges Hospital} Medical School, Tooting, London, UK

Introduction: Neonatal neutrophils are thought to be deficient compared to adult, especially at times of stress. To investigate sensitivity of respiratory burst to stimulation neutrophils from adults, healthy neonates (HN) and stressed neonates (SN) were stimulated with serially increasing concentrations of PMA. Changes in granule production and cell size were also recorded.

Method: A whole blood technique of flow cytometry was used to determine respiratory burst. Adult $(n=10), H N(n=10)$ (median gestation (age) $=35 w k$ $(8 d))$ and $S N(n=8)$ (median gestation (age) $=27 w k(19 d))$ samples were tested simultaneously. Changes in side scatter and forward scatter properties of the neutrophil populations were recorded as measures of granularity and cell size, respectively.

Results: Median fluorescence (milli-equivalents of fluorecscence) following maximum stimulation were adult $=16.4, \mathrm{HN}=63$ ( $\mathrm{p}<0.01), \mathrm{SN}=99.7$ ( $\mathrm{p}<0.01)$. Resting fluorescence (without stimulation) of each population were not significantly different. Regression coefficients (slope; $b$ ) of fluorescence for increasing PMA stimulation were adult=1.09 $(\mathrm{p}<0.001), \mathrm{HN}=1.19(\mathrm{p}<0.001)$ and $\mathrm{SN}=1.19(\mathrm{p}<0.001)$. Both neonatal lines of regression were significantly different from adult $(P<0.01)$. Granule production and discharge, as judged by granularity and size of the cell populations, occurred at lower concentrations of stimulant for neonates than adults.

Conclusion: By these measures neonatal neutrophils are more sensitive to stimulation with PMA than adult. This study's findings are contrary to reports that neutrophils from stressed neonates are less efficient than healthy neonatal or adult neutrophils.

\section{G181 PROSPECTIVE STUDY OF THE PREVALENCE OF LATEX ALLERGY IN CHILDREN REFERRED TO A SUPRAREGIONAL SURGICAL CENTRE}

Hourihane J, Allard J, Kurtianyk O, Gronlund $\mathrm{H}^{\dagger}$, McEwan A, Strobel S Institute of Child Health and Great Ormond St Hospital, London England, Pharmacia \& Upjohn Diagnostics, Uppsala, Sweden

Background: The prevalence of latex allergy is highest in children who have multiple operations, with rates of $60 \%$ reported in children with spina bifida and urogenital malformations. No prospective studies of the prevalence of latex sensitisation in children have been undertaken in Britain.

Method: We investigated the prevalence of latex allergy and sensitisation in children due for surgery at Great Ormond Street Hospital using a questionnaire based on the ISAAC questionnaire. Total IgE and latex-specific IgE were measured with the UniCAP system $B$.

Results: 255 subjects, 160 males. $127(49.8 \%)$ gave a history of atopic disease. Median current age was 47 months and median number of operations was 4 . Fifty subjects $(20 \%)$ had $>5$ previous operations. Seventy-seven subjects had total IgE above normal for their age (35 with atopic history, 42 without). Two subjects gave a convincing positive history of reactions to latex. Both had raised latex-specific $\operatorname{lgE}$, giving a prevalence of latex allergy in this paediatric surgical population of $0.8 \%$. Four other subjects had raised latex-specific IgE but had not reported clinical reactions to latex. The prevalence of asymptomatic latex sensitisation is $1.6 \%$. The combined prevalence of latex allergy and latex sensitisation is $2.4 \%$. Five of the six subjects with raised latex $\operatorname{lgE}$ had a clinical diagnosis at first presentation that would have predicted multiple surgical procedures. The prevalence of latex allergy or latex sensitisation in subjects with $>5$ previous operations is $5 / 50(10 \%)$. Comparing the 6 latex allergic or sensitised subjects with the total group of 255 , they were older (median age 12.75 years, $p=0.007$ ), and had more operations (median 18, $\mathrm{p}<0.022$ ).

Conclusions: Latex allergy affects $0.8 \%$ of the highly selected population studied, but twice that number are sensitised to latex. The overall prevalence of latex allergy or sensitisation is $2.4 \%$. A policy to minimise latex sensitisation of children needs to be developed.

\section{G182 DEVELOPMENT OF IMMUNE TOLERANCE IN PEANUT ALLERGY}

SA Connolly, S Lade, PB Sullivan, C Robertson, M Elawad John Radcliffe Hospital, Department of Paediatrics, Oxford

Many children have been unnecessarily prescribed self-injectable adrenaline for peanut allergy previously considered to be life long. Little is known of the development of immune tolerance to peanut antigens. This study aimed to seek evidence of immune tolerance by direct challenge in peanut allergic children over 5 years of age. 13 children were selected, all had had their first reaction at $<4$ yrs. All were carrying adrenaline although none had used it. None had had an anaphylactic reaction but symptoms varied from tingling of the tongue with swollen lips, to facial swelling and wheeze.
Under close in-patient medical supervision and with an I.V cannula in situ, a skin prick test was performed followed by a staged oral challenge with peanut butter.

$5 / 13$ children had no reaction on skin prick testing, $3 / 13$ had a weal of $<$ $64 \mathrm{~mm}^{2}, 5 / 13>64 \mathrm{~mm}^{2}$. Of these, 12 were challenged orally, $7 / 12$ had a negative result and were able to discontinue carrying adrenaline.

In this group of selected children with relatively mild reactions, no evidence of anaphylaxis and whose reactions had been at a relatively early age , 54\% appeared to have developed immune tolerance. All children that had a successful challenge, had a weal of $<64 \mathrm{~mm}^{2}$ These children were able to discontinue carrying their adrenaline. No child had a severe reaction during the oral challenge.

\section{G183 LIVER DISEASE IN CHILDREN WITH HYPER IGM SYNDROME}

F Rodrigues ${ }^{1}$, N Hadzic ${ }^{1}$, G Davies ${ }^{2}$, A Jones ${ }^{2}$, P Veys ${ }^{2}, \mathrm{~J} \mathrm{McLauchlin}^{3}, \mathrm{G}$ Mieli-Vergani ${ }^{1}{ }^{1}$ Department of Child Health, King's College Hospital, ${ }^{2}$ Host Defence Unit, Great Ormond Street, and ${ }^{3}$ Central Public Health Laboratory, Colindale, London, UK

Hyper IgM syndrome is an immunodeficiency caused in most cases by an Xlinked deficiency of CD40 ligand (CD40L) expression. The patients have lifethreatening infections in infancy, may become colonised with Cryptosporidium parvum $(C p)$ and develop sclerosing cholangitis (SC). To determine liver involvement and outcome of patients with hyper IgM syndrome, we reviewed retrospectively 20 children (all boys), from 16 families, referred to us between 199099. Seventeen had CD40L deficiency confirmed by genetic studies. Patients were assessed by liver function tests (LFTs) (20), ultrasound scan (USS) (20), liver biopsy (19), ERCP (17) and Cp screening by microscopy and PCR in the stools (17) and bile (7). SC was diagnosed in 9 patients (45\%) based on ERCP and histology (5), ERCP (3), and histology (1). Five children had severe, 1 mild and 2 minor radiological changes. USS was abnormal in 6/9 $(67 \%)$ patients with SC. Of the 13 (65\%) with normal LFTs, 2 had minor and 2 mild cholangiopathy. All children with persistently abnormal LFTs (5) (median AST $117 \mathrm{IU} / \mathrm{/}$, range: 77-142; GGT 182 IU/l, range: 128-299) had abnormal USS, liver biopsy and ERCP. $1 / 7$ bile and $5 / 17$ stool specimens tested were $C p$ positive. Four $C p$ positive patients have SC. Four patients had bone marrow transplant (BMT) [1 non-myeloablative $(\mathrm{nm})]$ and 1 combined liver transplant (LT) and nm-BMT. Two boys died of disseminated $C p, 1$ after conventional BMT and urgent LT for liver failure, the other after re-LT. The boy, $C p$ negative, who had combined LT and nm-BMT has normal immune and liver function 1 year later. In conclusion, children with hyper IgM syndrome and persistently abnormal LFTs have established cholangiopathy. If colonised with $C p$ their outcome after BMT and LT is uncertain. BMT should be considered before severe liver damage. Combined LT and nm-BMT should be attempted in advanced liver disease.

\section{G184 DETECTION OF LIVER KIDNEY MICROSOMAL TYPE 1} ANTIBODY USING MOLECULARLY BASED IMMUNOASSAYS

Kerkar $\mathrm{N}^{1,2}$, Davies $\mathrm{E} \mathrm{T}^{3}$, Williams $\mathrm{R}^{1}$, Mieli-Vergani $\mathrm{G}^{2}$ \& Vergani $\mathrm{D}^{1}$ ${ }^{1}$ Institute of Hepatology, University College London Medical School, ${ }^{2}$ Department of Child Health and ${ }^{3}$ Immunology, King's College Hospital, London, UK

Liver kidney microsomal antibody type 1 (LKM1), the hallmark of autoimmune hepatitis type $2(\mathrm{AlH} 2)$ is often mistaken for antimitochondrial antibody when it is detected by the conventional technique of immunofluorescence [(IFL), (Gastroenterology 1992; 103: 1290)]. LKM1 is also found in up to $10 \%$ of patients with chronic HCV infection, where its presence may be associated to serious side effects during Interferon treatment (Gut 1999; 45: 440-441). The molecular target of LKM1 has been identified as cytochrome P450 2D6 (CYP2D6) enabling development of immunoassays detecting anti-CYP2D6 antibodies. Using IFL as reference, we measured anti-CYP2D6 with an inhouse ELISA ( $\mathrm{J}$ Immunol Meth 1999; 223:227) and 2 new commercial immunoassays (Varelisa and $M B L$ ) in 31 patients with AlH2 - all positive at diagnosis for LKM1 - obtained at different stages of their disease. Controls were 29 patients with $\mathrm{AlH} 1,8$ with $\alpha-1$ antitrypsin deficiency, 8 with Alagille's syndrome, and 10 healthy children. Twenty nine of the 31 sera from AlH2 were positive by IFL and by the in-house ELISA, 28 by Varelisa and by MBL. All 53 sera negative by IFL were also negative by the in-house ELISA and Varelisa, while 11 marginally exceeded the upper limit of normal indicated by the manufacturers for the $M B L$ assay, though their absorbance values were significantly lower than those of the positive. Kappa values for the in-house ELISA, the Varelisa and MBL when compared with IFL were 0.95, 0.87 and 0.76 respectively, indicating a high degree of concordance. These data show that the two available commercial immunoassays are reliable for the measure of LKM1. Their use should abolish one of the most frequent errors of the immunological diagnostic laboratory. 


\section{G185 BEHAVIOURAL AND PSYCHOLOGICAL ABNORMALITIES IN} ADENOSINE DEAMINASE DEFICIENCY

Rogers $\mathrm{M}^{\star}$, Lwin $\mathrm{R}^{\star}$, Gaspar $\mathrm{HB}^{\dagger}{ }^{\star}$ Behavioural Sciences Unit, ${ }^{\dagger}$ Molecular Immunology Unit, Institute of Child Health, University College London, London WC1N 1EH

Adenosine Deaminase (ADA) deficiency is an inherited disorder that results in abnormalities of the immune system leading to severe combined immunodeficiency (SCID). In severely affected patients, death occurs in the first year of life. Successful immunological correction is now available for ADA-SCID patients by means of bone marrow transplantation (BMT). However non-immunological dysfunction remains even after BMT, notably psychological and behavioural abnormalities have been observed, though not previously investigated in a controlled manner.

Independent matched pairs were drawn from the outpatient population of Great Ormond Street Hospital. Eleven pairs of ADA-SCID patients and nonADA-SCID transplanted patients were matched for gender, age, age at transplant and type of transplant. Psychological assessment and psychometric tests were administered to evaluate and document the behavioural, psychologica and neuro-developmental functioning in ADA-SCID patients after BMT.

Results indicate the ADA-SCID group functioning in the abnormal/pathological range on all measured socio-behavioural domains; mean behavioura scores for the control group were all within normal limits. Paired t-tests indicated significant differences $(p<.05)$, with large effect sizes, in the domains of social behaviour and hyperactivity and large differences across other behavioural domains. General cognitive ability (below average for both groups) and educational attainment were not different between the ADA-SCIDs and controls.

These findings suggest that ADA-SCID patients experience significant behavioural difficulties even after treatment and this may reflect the natural history of the condition now that survival is assured through BMT. Further assessment of other UK and European ADA deficient children is planned.

\section{G186 UK EXPERIENCE OF BONE MARROW TRANSPLANTATION FOR CD40 LIGAND DEFICIENCY}

Khawaja $K^{\star}$, Cant $A J^{\star}$, Veys $\mathrm{P}^{\circ}$, Kelly $D A^{\infty}$, Hadzic $\mathrm{N}^{\bullet}$, Jones $\mathrm{AM}^{\circ}$, Pagliuca $\mathrm{A}^{*}$, Hodges $\mathrm{S}^{*}$, Davies $\mathrm{EG}^{\circ}$ Newcastle General Hospital Newcastle upon Tyne*, Great Ormond Street Hospital - London ${ }^{\circ}$ Birmingham Children's Hospital Birmingham ${ }^{\infty}$, King's College Hospital London"

CD40 Ligand deficiency (CD40LD) is an X linked disorder of primary immunodeficiency resulting in failure of immunoglobulin isotype switching as well as susceptibility to intracellular pathogens including Cryptosporidium parvum (CP) infection. European data shows only $20 \%$ survival of affected boys beyond the age of 25 years. Liver disease, including sclerosing cholangitis which may be $\mathrm{CP}$ associated, is a major determinant of mortality. Attempted cure by BMT was performed in 11 boys of median age 10 years (range 3-18) in two UK centres. Matched sibling donors were used in 4, fully matched donor in 3 and one antigen mismatched unrelated donor in 4 . Conditioning regimens were Busulphan/Cyclophosphamide (7), total body irradiation / cyclophosphamide (1) or a non myeloablative regimen (Fludarabine / Melphalan) in 3. Six had sclerosing cholangitis including one with cirrhosis and chronic pancreatitis. 4 had known previous or current cryptosporidial infection. One patient underwent elective liver transplant prior to BMT.

Five patients (4 with pre-existing liver disease) died: three from progressive cryptosporidial infection and liver inflammation, one from GvHD and disseminated adenovirus infection and one from severe GvHD. Six patients are alive and well with normal immune function including 4 with no pre-existing live disease and 2 with liver disease who received the non myeloablative regimen (follow up 5-30 months).

Younger age, absence of cryptosporidium and normal liver histology was associated with better outcome. Non myeloablative conditioning regimen may be associated with a better outcome in the higher risk patients.

\section{G187 MANAGEMENT OF A HOSPITAL OUTBREAK OF INVASIVE ASPERGILLOSIS IN IMMUNOCOMPROMISED AND ICU PATIENTS ASSOCIATED WITH INCREASED ENVIRONMENTAL EXPOSURE}

Crooks BNA, Galloway A, Flood TJ, Skinner R, Hale J, Verrill M, Fulton B, Poplett A Newcastle General Hospital - Newcastle upon Tyne

Aspergillus is an ubiquitous environmental organism, only causing significant disease in immunocompromised or sick patients. Mortality from invasive aspergillosis (IA) ranges from $30 \%$ in AIDS patients to over $90 \%$ in neutropenic BMT patients. Incidence is increasing due to increases in transplantation programmes, aggressive chemotherapy regimens and HIV disease. A source of infection is often not identified, and many cases may result from activation of endogenous colonisation. Prevention of cases relies on identification of at-risk patients, adequate antifungal prophylaxis and avoidance of exposure to airborne spores. We report a cluster of 7 cases of proven and suspected IA, fatal in $3 / 7(43 \%)$ where increased exposure to building dust from a large-scale onsite demolition project may have been a significant factor. The first patient was admitted with meningococcal septicaemia, developed bowel perforation due to Aspergillus fumigatus and died. A second case of IA in an adult PBSCT autograft patient was diagnosed 2 weeks later and also died. A multidisciplinary infection control team advised stopping demolition after identification of case 2. 5 further cases of definite and probable IA (1 PICU patient, allogeneic BMT (died), 1 PBSCT autograft patient and 2 oncology patients receiving chemotherapy) were subsequently diagnosed. Heavy aspergillus contamination was found in all ward areas and air conditioning systems sampled, except cubicles with HEPA filtration or laminar air flow. Additional measures to interrupt spore transmission included sealing windows and ducts, increased cleaning, partitioning of internal building work and removal of plants and flowers from clinical areas. Positive pressure HEPA filtration ventilation was installed in additional key areas. Surveillance using air sampling techniques demonstrated a reduction in spore counts after these actions. Evidence for an environmental source of aspergillus included historically low rates of IA (1 in the previous year), temporal clustering of cases and lack of further cases after demolition ceased. Further demolition is due to start after these measures are in place. Environmental and clinical monitoring will continue.

\section{G188 DETECTION OF CRYPTOSPORIDIUM BY POLYMERASE CHAIN REACTION (PCR) IN PATIENTS WITH PRIMARY IMMUNODEFICIENCIES}

Davies EG, Amar C, Hadzic $\mathrm{N}^{\star}$, McLauchlin J Food Safety Microbiology Laboratory, PHLS Central Public Health Laboratory, London; * Paediatric Liver Unit, Kings College Hospital; Dept of Immunology, Great Ormond Street Hospital, London

Cryptosporidium parvum (Cp) is associated with chronic infection of the biliary tree leading to sclerosing cholangitis in patients with immunodeficiency disorders. The conventional method of diagnosis involves microscopic detection of oocysts. It is possible that asymptomatic carriage of $\mathrm{Cp}$ occurs below the limits of detection of these tests. This study set out to assess the role of PCR in the detection of $\mathrm{Cp}$ gene sequences in patients with primary immunodeficiencies.

Samples $(n=28)$ from 15 children with: CD40 ligand deficiency (6); unspecified combined immunodeficiency (7); MHC Class II deficiency (1) and interferon $\mathrm{c}$ receptor deficiency (1) were examined by light microscopy (following staining by modified Ziehl Neelsen and immunofluorescence) and by using three different PCR procedures: one for cryptosporidium outer wall protein gene (COWP) and two different 18S rDNA sequences. Samples included 14 stool, 8 bile, 5 liver tissue and 1 urine. Four samples only (from 2 patients) were collected during episodes of diarrhoea. The urine and all liver samples were negative by all tests. Cp was detected in 2/14 stools (both collected during diarrhoeal episodes) by both microscopy methods and by all three PCR procedures. All other samples were negative by microscopy, but one of the 18S rDNA sequences was detected in one further stool and one bile sample. COWP gene sequences were detected in a further 5 stools (including the additional one positive by $18 \mathrm{~S}$ rDNA PCR) and in 2 bile samples.

Detection of $\mathrm{Cp}$ nucleic acid by PCR is more sensitive than microscopy when applied to asymptomatic children with primary immunodeficiencies. Detection of COWP fragments was the most sensitive method used here.

\section{G189 STUDIES ON THE VACCINE CANDIDACY OF INNER CORE LIPOPOLYSACCHARIDE (LPS) IN NEISSERIA MENINGITIDIS (NM) GROUP B}

Plested $\mathrm{JS}^{1,2}$, Makepeace $\mathrm{KM}^{1}$, Coull $\mathrm{P}^{1,2}$, Mackinnon $\mathrm{FM}^{1}$, Griffiths $\mathrm{HG}^{2}$, Gidney MA ${ }^{3}$, Lacelle $\mathrm{S}^{3}$, Cox $\mathrm{AD}^{3}$, Richards $\mathrm{JC}^{3}$, and Moxon ER ${ }^{1}$ Department Paediatrics, University of Oxford: ${ }^{2}$ Department Clinical Immunology, Oxford: ${ }^{3}$ NRC, Ottawa, Canada

Aims: To identify conserved, accessible and potentially cross-reactive $\mathrm{Nm}$ serogroup B LPS epitopes that may be potential vaccine candidates.

Methods: Previously we described an inner core LPS epitope that was accessible and conserved in $70 \%$ of a global collection of $100 \mathrm{Nm}$ strains representative of all major serogroups [Plested et al., 1999 IAI 67: 54175426]. The conserved epitope recognised by monoclonal antibody (mab) B5 was identified in all LPS immunotypes with phosphoethanolamine (PEtn) in the 3-position of $\beta$-chain heptose (Hepll) of inner core LPS. Murine mabs to immunotype L4 (PEtn in 6/7 position) obtained using formalin-fixed $\mathrm{Nm}$ L4 galE whole-cells were screened by ELISA against purified LPS from $\mathrm{Nm}$ mutant and wild-type strains. Conservation and accessibility was assessed by: (1) dot blots of whole-cell lysates of $N m$; (2) immunofluorescence microscopy.

Results: One of the mabs, A4 (lgG2 $)$, demonstrated specificity for both L4 galE and L2 galE LPS and recognised all except $3 \mathrm{Nm}$ mab B5- strains. Together mabs B5 and A4 recognise 97/100 Nm strains. Mab A4 accesses the inner core epitope in the L4 galE mutant in the presence of capsule.

Conclusion: Inner core glycoforms have been identified with PEtn in either (i) 3-position of Hepll (mab B5), (ii) 6/7- position of Hepll (mab A4) or (iii) absent. These studies indicate that strains of $N m$ may possess at least 3 nner core epitopes, findings that support the feasability that inner core LPS may have potential as a $\mathrm{Nm}$ Group B vaccine. 
G190 OPTIMISING INVESTIGATIONS IN MENINGOCOCCAL DISEASE-A HOSPITAL BASED RETROSPECTIVE ANALYSIS

KC Khoobarry, J Alexander North Staffordshire Hospital, Stoke-on-Trent

Enhanced surveillance of meningococcal disease was started in 1998 for future evaluation of impact of vaccination. Recommended investigations include blood culture, PCR, throat swab, serology, CSF culture and PCR and skin scraping if appropriate. An audit at North Staffordshire hospital in 1997 and a similar study in Birmingham showed laboratory confirmation of meningococcal disease was less than $60 \%$ and $42 \%$ respectively. We therefore implemented recommended guidelines to optimise investigations and improve laboratory confirmation.

Aims: To determine the proportion of cases of clinically diagnosed meningococcal disease that are investigated appropriately after implementation of guidelines and the proportion of cases that are confirmed by various laboratory methods.

Methods: Retrospective case notes review of all children aged 0-16 years admitted between January-December 1998 with clinically diagnosed meningococcal disease. All investigations performed during admission and subsequent follow-up were analysed.

Results: 40 children were admitted with meningococcal disease; $32(80 \%)$ were investigated according to recommended guidelines.

$30 / 40(75 \%)$ cases had diagnosis confirmed by laboratory methods. Laboratory confirmation was $94 \%$ (30/32) in the group who had all investigations performed according to recommended guidelines.

Conclusion: The proportion of laboratory confirmed cases improved after implementation of guidelines and was significantly higher when all recommended investigations were performed to optimise laboratory confirmation. There needs to be an increased awareness of recommended guidelines for investigation of clinically suspected meningococcal disease.

\section{G191 THE ROLE OF RANTES IN MENINGOCOCCAL DISEASE}

ED Carrol, APJ Thomson, KJ Mobbs*, CA Hart* Institute of Child Health, Royal Liverpool Children's Hospital NHS Trust, Alder Hey, Eaton Road, Liverpool, L12 2AP, UK; * Department of Medical Microbiology, $8^{\text {th }}$ Floor Duncan Building, University of Liverpool, Daulby Street, Liverpool L69 3GA, UK

Introduction: The chemokine RANTES (Regulated on Activation Normal T Cell Expressed and Secreted) is a potent regulator of leucocyte trafficking RANTES has been shown to play an important role in directing the migration of monocytes, memory T lymphocytes, eosinophils, basophils and natural killer cells. It preferentially attracts mature CD4 cells as well as macrophages and eosinophils, but not neutrophils. Its role has not previously been described in meningococcal disease (MCD)

Aims: This study aimed to determine the role of RANTES in the pathophysiology of MCD

Methods: 165 children with MCD were prospectively studied. Plasma RANTES, IL-8, IL-6, IL-1Ra and TNF-a were measured by an enzyme amplified sensitivity immunoassay (EASIA) on admission. Severity of disease was stratified by the Glasgow Meningococcal Septicaemia Prognostic Score (GMSPS).

Results: RANTES levels correlated significantly with IL-8 levels $(r=-0.36$, $p<0.0005)$, GMSPS $(r=-0.36, p<0.0005)$, admission lactate levels $(r=-0.26$, $p=0.0001)$, platelets, prothrombin time $(r=-0.32, p=0.001)$, and APPT $(r=-0.26$, $\mathrm{p}=0.006$ ). RANTES levels were lower in those with severe disease (GMSPS $>8$ ) than those without (GMSPS $<8$ ), $p=0.001$, in those with septic shock than in those without, $p<0.0005$, and in non-survivors than in survivors, $p=0.048$, Mann Whitney. There was no significant difference in RANTES levels between disease types; meningitis (MM), mixed meningitis/septicaemia (MM/MS) and septicaemia (MS).

Conclusions: RANTES is a potential mediator in the pathophysiology of MCD. Its chemoattractant properties for T cells and monocytes appear to be beneficial in modulating the effects of pro-inflammatory cytokines such as TNF$\alpha$.

\section{G192 INTERLEUKIN-6 GENE POLYMORPHISMS IN MENINGOCOCCAL DISEASE}

Carrol ED, Mobbs KJ *, Thomson APJ, Hart CA* Institute of Child Health, Royal Liverpool Children's Hospital NHS Trust, Alder Hey, Eaton Road, Liverpool L12 2AP, *Department of Medical Microbiology, University of Liverpool, Duncan Building, Daulby Street, Liverpool L69 3GA

Introduction: Interleukin-6 (IL-6) is an important mediator in the pathophysiology of meningococcal disease (MCD). High levels of this cytokine are associated with more severe disease. Genetic factors are known to play an important role both in susceptibility to MCD and risk of severe disease. The aim of this study was to determine if polymorphisms in the IL-6 gene are associated with increased susceptibility to disease or increased risk of severe disease or death.

Methods: 127 children with meningococcal disease were prospectively studied. Severity of disease was stratified using the Glasgow Meningococcal Septicaemia Prognostic Score (GMSPS). Genomic DNA was extracted and a 200bp region of the IL- 6 gene was amplified by polymerase chain reaction (PCR). After restriction digestion, a G/C restriction fragment length polymorphism (RFLP) was identified at position -174 of the IL-6 gene. 99 healthy adult blood donors were used as controls.

Findings: Three genotypes were identified; G/G, G/C and C/C. The frequency of the $\mathrm{C}$ allele was $0.307(95 \% \mathrm{Cl} 0.25-0.36)$ in patients, compared to 0.444 (95\% Cl 0.38-0.51) in healthy adult controls. A higher proportion of those with the $\mathrm{G} / \mathrm{G}$ genotype had MCD compared to the control population, $\chi^{2}=11.011, p=0.001$. Possession of the $C$ allele was not associated with more severe disease (GMSPS $>8$ ) or death.

Conclusion: The lower frequency of the $C$ allele in patients with MCD suggests that possession of this allele may confer protection against the development of disease. It does not appear to determine disease severity or risk of death.

\section{G193}

\section{WITHDRAWN}

\section{G194 SEQUENCING ANTIRETROVIRAL THERAPY (ART) IN THE SOUTH LONDON PAEDIATRIC HIV COHORT}

\section{Doerholt K, Sharland M, Ball C, du Mont G PHILS-NET (Paediatric HIV in} London South Network)

Background: Following the introduction of combination ART in 1997, 13 drugs are now available. No audit data is available on the paediatric use of ART outside clinical trials demonstrating their impact on standard outcome measures.

Objectives: To audit paediatric ART use in south London.

Design: Retrospective notes review.

Cohort: 101 children followed from January 1996 to September 1999

Measurements: demographic variables, ART use, VL (start/nadir/end), CD4 count (start/peak/end).

Results: 84 children $(83 \%)(47 \mathrm{M}$; $54 \mathrm{~F}$ - median $6.4 \mathrm{yrs})$ commenced combination ART, and have received 160 regimens. Following failure of first line ART regimen, children sequence onto $2^{\text {nd }}$ and $3^{\text {rd }}$ line regimens. The mean duration of completed regimens was $1^{\text {st }}$ line-356 days, $2^{\text {nd }}$ line $288-$ days, $3^{\text {rd }}$ line 281 days. The mean VL reduction was $1^{\text {st }}$ line $-1.53 \log _{10}, 2^{\text {nd }}$ line -0.85 $\log _{10}$, and $3^{\text {rd }}$ line $-0.83 \log _{10} .33 \%$ of the cohort reached an undetectable VL during $1^{\text {st }}$ line regimens compared to $22 \%$ during $3^{\text {rd }}$ line therapy. Mean CD4 count increase was $1^{\text {st }}$ line $287,3^{\text {rd }}$ line 200 . Regimens were changed due to clinical $-6 \%$, immunological $-6 \%$, toxicity $19 \%$, virological $-60 \%$, or adherence $25 \%$ failure.

Conclusion: Children with HIV rapidly sequencing through ART regimens. The duration and impact of each regimen decreases with each change. it is unclear whether new drug development will keep pace with current drug use. A more cautious approach to drug sequencing following virological failure may be appropriate. 


\section{G195 ONE YEAR EXPERIENCE OF NELFINAVIR IN UK PAEDIATRIC HIV-1 COHORT}

Z Mitchla ${ }^{1}$, M Sharland ${ }^{1}$, N Delmas ${ }^{2}$, D Gibb ${ }^{3}$, G Tudor-Williams ${ }^{4}$, C Ball ${ }^{4}, \mathrm{G}$ Dumont ${ }^{4}$, N Klein ${ }^{4}$ St George's Hospital, London UK; ${ }^{2}$ Roche UK; ${ }^{3} M R C$ London UK; ${ }^{4}$ Paediatric HIV London Interest Group

Objective: To audit the long-term clinical experience of nelfinavir (NFV) in children with HIV-1 infection in the UK

Method: Retrospective multi-centre observational analysis of CD4 and viral load $\left(V_{\mathrm{L}}\right)$ measurements in HIV-infected children enrolled into the NFV named patient programme.

Results: Fifty-five HIV-infected children (age $6 \mathrm{~m}$ to 16 yrs: median 6 years) who had completed at least 12 month follow-up were analysed. 36/55 (66\%) were CDC clinical category C. $76 \%$ were antiretroviral experienced but only $7 \%$ PI treated. NRTI combinations used with NFV were d4T/3TC (53\%), AZT/dd $(16 \%)$ and $\mathrm{d} 4 \mathrm{~T} / \mathrm{ddl}(13 \%)$. In $18(33 \%)$ NFV was added to existing NRT regime. Mean dose of NFV was $70.8 \mathrm{mg} / \mathrm{Kg} /$ day (range: 41 to 98.7 ). Median CD4 counts were 164 cells $/ \mathrm{mm}^{3}$ (baseline), 388(3m), 611(6m) and $648(12 \mathrm{~m})$ showing an average 2.8-fold rise from baseline to 12 months. Median $V_{L}$ was $5.04 \log _{10}$ (baseline), $3.54 \log _{10}(3 \mathrm{~m}), 3.6 \log _{10}(6 \mathrm{~m})$ and $3.9 \log _{10}(12 \mathrm{~m})$. The proportion of children with undetectable $\mathrm{V}_{\mathrm{L}}$ (defined as $<400 \mathrm{copies} / \mathrm{ml}$ ) at 3,6 and 12 months was 33,31 and $24 \%$ respectively. In a multiple regression analysis, younger age $(p=0.003)$, higher NFV dose $(p=0.03)$ and change of NRTI at commencement of NFV were associated with better CD4 response. There was also a trend for higher doses of NFV to produce greater $V_{L}$ reductions. $43 / 55(78 \%)$ of children remained on full dose NFV at 12 months. Of the 12 who withdrew, 6 were due to treatment failure, 2 refused to take NFV and child stopped due to side effects, 1 died and 2 left the UK.

Conclusion: The one-year analysis of this cohort demonstrates that NFV remains well tolerated, with continued CD4 improvement, but a significant rise in $V_{L}$ from nadir. Despite virological failure most of the children in the UK have not changed therapy.

\section{G196 THE SIGNIFICANCE OF FEVER IN CHILDREN}

Nademi Z, Clark JE, Cant AJ Paediatric Immunology and Infectious Diseases Unit, (The Newcastle upon Tyne Hospitals NHS Trust) Newcastle General Hospital, Westgate Road, Newcastle upon Tyne

Fever is a common presenting symptom in casualty. Differentiating the serously ill is difficult; previous studies have suggested that symptoms, signs and simple investigations may be helpful.

Aims: to assess causes of fever and identify clinical and laboratory features suggesting serious illness in children presenting with temperatures over $38^{\circ} \mathrm{C}$ then to establish clinical guidelines.

Methods: for 3 months (August October 1999) all children with a temperature of $\geq 38^{\circ} \mathrm{C}$ seen in two hospitals were assessed.

Results: 142 children were seen, $64 \%$ male, $51 \%$ aged 3 months -2 years $80 \%$ had temperatures between $38^{\circ} \mathrm{C}-39^{\circ} \mathrm{C}$. $95 \%$ were GP referrals and $5 \%$ were tertiary referrals. Serious infection was present in $24 \%(34)-17 \%$ (24) microbiologically or radiologically proven; meningitis (7), sepsis (5), brain abscess (2), toxic shock (1), pneumonia (9). $20 \%$ (27/135) of GP referrals had serious infection. $42 \%(5 / 12)$ of GP referrals had serious infection. $42 \%$ $(5 / 12)$ of microbiologically proven meningitis and sepsis and $36 \%(8 / 22)$ of all meningitis and sepsis were meningococcal. $77 \%$ had non serious infections appendicitis (2), AOM (4), tonsillitis (12), gastroenteritis (8), febrile convulsion (16), UTI (5), abscess (1), stomatitis (1), lymphangitis (2), cellulitis (3), irritable hip (1), conjunctivitis (1) and non-specific with unknown aetiology $(51 \cdot 36 \%)$. In cases of proven serious infection the temperature was $38^{\circ} \mathrm{C}-38.5^{\circ} \mathrm{C}$ in $42 \%$, $38.6^{\circ} \mathrm{C}-39^{\circ} \mathrm{C}$ in $46 \%, 39.1^{\circ} \mathrm{C}-39.5^{\circ} \mathrm{C}$ in $8 \%$ and $39.6^{\circ} \mathrm{C}-40^{\circ} \mathrm{C}$ in $4 \% .22 \%$ had received antibiotics, $22 \%$ had a rash. $42 \%$ of the non-serious group and $10 \%$ of the serious group had a history of poor feeding, history of vomiting was in $30 \%$ and $9 \%$ respectively. FBC was taken in $48 \%$ of patients; in $4 \%$ of serious and $10 \%$ non serious infections WBC was $<5000$, and it was between $5000-15000$ in $52 \%$ and $54 \%$ respectively.

Conclusions: $1 / 5$ of children referred with fever had serious infections. Clinical signs and laboratory investigations did not distinguish serious from non-serious infections. Guidelines will not replace clinical assessment of the child with a temperature.

\section{G197 ACYCLOVIR THERAPY FOR VENTILATED CHILDREN WITH SUSPECTED HSE-WHO? WHEN? HOW LONG?}

Mehta N, Karnik L, Walters S, Brown D, Watkins R, Levin M, Britto J PICU, Department of Paediatrics, St Mary's Hospital, London W2

Aims: To review the presentation and investigations of ventilated patients started on acyclovir for suspected Herpes Simplex Encephalopathy (HSE) To propose an algorithm for management of this subset of patients.

Methods: We reviewed patients, ventilated for neurorespiratory failure over a period of 36 months, in whom acyclovir was started on clinical suspicion of $\mathrm{HSE}$, prior to PICU admission. Each patient was investigated for HSE, follow ing an algorithm which consisted of; a) EEG, b) Herpes simplex virus (HSV) serology, c) CSF examination (including PCR for HSV DNA) and d) neuroimaging. Acyclovir was discontinued if bedside neurology was normal after extu- bation and cessation of sedation; with no abnormality on any of the above investigations. The rest were continued on acyclovir for upto 21 days. Acyclovir was stopped earlier (second week of treatment) if repeat investigations (a to d) and neurology were normal or if an alternative diagnosis was confirmed.

Results: 80 cases (age 2 weeks to 14 years), with encephalopathy, ventilated on PICU, received acyclovir. The commonest presentation was febrile encephalopathy with generalised seizures $(n=40)$ followed by isolated febrile encephalopathy $(n=25)$, poor feeding, irritability and apnoea $(n=10$, infants), focal seizures $(n=5)$ and cranial nerve palsy $(n=1)$. CSF PCR for HSV DNA was positive in 2 of the 59 cases tested. Commonest EEG abnormality $(n=14)$ was generalised slowing. 31 patients completed acyclovir therapy.

Conclusion: Antiviral therapy for suspected HSE is initiated on clinical suspicion. However, in sedated, ventilated children bedside neurology is unreliable and lumbar puncture may be contraindicated in the acute phase. Our algorithm, combining clinical and laboratory investigations may facilitate decisions regarding ongoing antiviral therapy in these patients.

\section{G198 THE MANTOUX TUBERCULIN TEST AND ITS RELATIONSHIP TO THE HEAF TEST, BCG, AND TUBERCULOSIS}

Julia E Clark, Andrew J Cant Department Paediatric Immunology and Infectious Diseases, Newcastle General Hospital, Newcastle, NE6 4BE

Introduction: The Mantoux test is one of the oldest diagnostic tests still in use, but its correlation with the Heaf test, interpretation after previous BCG and use in detecting tuberculosis in inner city UK children today is unknown.

Aims: To correlate size of Mantoux with the above.

Methods: Heaf grade, BCG status and Mantoux size were recorded in children found to be Heaf positive at school pre BCG screening and in those screened as contacts of tuberculosis between 1997 and 1999 (27 months), and children with tuberculosis from 1991-99.

Results: 275 school children seen. Of 222 without prior BCG, 195/222 had Heaf II of whom $48 \%$ gave a Mantoux of $<5 \mathrm{~mm}, 26 \% 5-14 \mathrm{~mm}, 25 \% \geq 15 \mathrm{~mm}$ $27 / 222$ had Heaf III/IV; $89 \% \geq 15 \mathrm{~mm}, 33 \% \geq 20 \mathrm{~mm}$. Of 53 with BCG, $11 / 53$ had Heaf II, of whom none gave a Mantoux < 5mm, 46\% 5-14mm, $54 \%>$ $15 \mathrm{~mm}$. $42 / 53$ had Heaf III/IV with $95 \%>15 \mathrm{~mm}, 71 \%>20 \mathrm{~mm}$. 111 contacts were seen, 59 without prior BCG, in whom Mantoux was 0mm in $58(98 \%)$. Of 52 with BCG 24/52 (46\%) had a Mantoux of $0 \mathrm{~mm}, 19 \% 5-9 \mathrm{~mm}$ and $35 \%$ $10 \mathrm{~mm}$. In children with tuberculosis, $20 \%(7 / 35)$ were Mantoux negative at $0 \mathrm{~mm}$ ( 3 of whom had meningitis), $3 \% 6-9 \mathrm{~mm}, 74 \%>10 \mathrm{~mm}, 43 \% \geq 15 \mathrm{~mm}$ and $31 \% \geq 20 \mathrm{~mm}$.

Conclusions: $48 \%$ of Heaf II were Mantoux negative, $25 \%$ significant. Increasing Heaf grade correlates with increasing Mantoux size. BCG does not change Mantoux size in $50 \%$, but in $50 \%$ increases size between 5 and $10 \mathrm{~mm}$. Mantoux can be negative in tuberculosis in about $20 \%$, although in most responses are $>/ 10 \mathrm{~mm}$.

\section{G199 DO WE REALLY NEED TO SEND STOOL SPECIMENS IN CHILDREN WITH GASTRO-ENTERITIS?}

Hamed H, Sinha GP Paediatric Department, Manor Hospital, Walsall, WS2 9PS

Introduction: Gastro-intestinal infection is one of the leading causes of morbidity and mortality worldwide and is caused by a wide variety of entero-pathogens, including bacteria, viruses and parasites. Most episodes are self-limited with low diagnostic yield and very few need antimicrobial therapy.

Aim: Our aim was to ascertain whether stool samples should be sent for examination in all cases of gastro-enteritis.

Method: We collated data from the Microbiology Department on all children under 16 from whom stool cultures were sent over a four year period (January, 1996 to November,1999) and from those patients with positive bacterial infection and cryptosporodia.

Results: The total number of specimens sent $=20,173(3,284$ for rotavirus, 2,300 for ova, cysts, parasites and 14,589 for culture and sensitivity. $16 \%$ were positive for rotavirus (523 out of 3,284 ), $2 \%$ for giardia ( 48 out of 2,300 ) and $1 \%$ for other organisms (81 salmonella, 75 campylobacter, 16 shigella, 11 cryptosporidium and $7 \mathrm{E}$. coli). Amongst the last group 75 out of 190 were admitted Family history of gastro-enteritis was noted in $9 \%$, foreign travel in $5 \%$ and travel to farm $1 \%$. The duration of diarrhoea in this group was 3 days or less in 51 patients and more than 3 in 21 patients, $73 \%$ had pyrexia, $57 \%$ had pain in the abdomen, $52 \%$ had vomiting, $28 \%$ were dehydrated, $12 \%$ were toxic, $4 \%$ had bloody diarrhoea, $24 \%$ had abnormal biochemistry and $35 \%$ required intravenous fluids. We have spent $£ 600,000$ on stool specimens in the last four years (£12.19 per specimen).

Conclusion: Stool specimens should only be sent of patients with bloody or prolonged diarrhoea, immuno-compromised patients, travellers from endemic areas, those with a family history of diarrhoea, farm visitors, toxic patients and during outbreak of diarrhoea. 


\section{G200 EARLY NEONATAL BONE MARROW TRANSPLANT IN} SEVERE COMBINED IMMUNODEFICIENCY

Kane LC, Crooks BNA, Dickinson A, Flood TJ, Abinun M, Gennery AR, Can AJ The Newcastle upon Tyne Hospitals NHS Trust - Newcastle upon Tyne In utero BMT has been proposed as best treatment for SCID as only $50 \%$ mismatched BMT are successful. However in-utero BMT is feasible only when positive family history leads to antenatal diagnosis. We reviewed 13 cases of SCID, diagnosed at birth, and referred to our unit for BMT between 1989 and 1999 (7 T-B+ SCID, 3 T- B- and 3 adenosine deaminase (ADA) deficiency). Sixteen BMT's were performed, one patient having 2 BMT's and one 3 BMT's. These BMTs consisted of 8 T cell depleted (TCD) haplo-identical, parental, 3 TCD unrelated donor and 5 whole marrow ( 2 matched paternal, 2 sibling and 1 sibling cord). Seven patients were conditioned for TCD BMT: all had cyclophosphamide $(200 \mathrm{mg} / \mathrm{kg}$ ) and busulphan $(5$ with $8 \mathrm{mg} / \mathrm{kg}$, and two with $16 \mathrm{mg} / \mathrm{kg}$ ). Follow up post-BMT ranges from 67 days to 10 years (median 3 years 9 months). All are alive and well. Six suffered transient acute graft versus host disease (aGvHD), grades 1 to 2, and two had chronic GvHD (cGvHD), now resolved. One patient has been transplanted 3 times for poor $T$ and $B$ cell function and cGvHD, and 1 is due for top up BMT for inadequate $T$ cell function. Of 11 patients evaluable for $T$ cell function, 10 have good proliferations to mitogen in vitro. Seven have normal $B$ cell function, and are off intravenous immunoglobulin (IVIg); six remain on IVIg, three for insufficient B cell function and three are less than 1 year post BMT. Eight of eleven evaluable childre have normal neurodevelopment ( 1 has behavioural problems, 1 has slow motor development, 1 with cGvHD related problems).

Conclusion: Early postnatal BMT for SCID before infection occurs has an excellent outcome with full survival and very high levels of $T$ and $B$ cell function even after TCD BMT. In-utero BMT will have to demonstrate no risk of hidden GvHD and good B cell function, as well as $100 \%$ engraftment, to be preferential to early postnatal BMT.

\section{G201 KAWASAKI DISEASE: DO WE NEED FULL AHA CRITERIA FOR DIAGNOSIS?}

Jayachandran R, Kumar Y, Sills JA Royal Liverpool Children's Hospital NHS Trust, Alder Hey

Aim: To determine whether American Heart Association (AHA) criteria for diagnosis of Kawasaki disease (KD) are being met. To determine the prognostic factors from the clinical and laboratory data.

Method: 80 patients with KD admitted to our hospital between Jan 1995 and Nov 1999 were identified from a hospital database. Case notes were examined to obtain the clinical and laboratory data. 12 patients who came for a cardiology opinion from other hospitals were excluded from the study. Complete information was obtained on 45 patients.

Results: All the 45 patients received immunoglobulins (lg). $44 \%$ of them did not meet AHA criteria at diagnosis and start of treatment. Duration of illness before Ig therapy was 9.3 days in those who met the AHA criteria and 7.6 days in those who did not meet the criteria. In infants, $87 \%$ (7 out of 8 ) had irritability and $63 \%$ (5 out of 8 ) had cardiac involvement. In the remaining children, $72 \%$ had irritability and $27 \%$ had cardiac involvement. All infants and $90 \%$ of children between 1 and 8 years of age with cardiac involvement showed irritability at presentation. While $80 \%$ of infants with incomplete AHA criteria had cardiac involvement, only $20 \%$ in the 1-8 year age group with incomplete criteria had cardiac involvement.

Conclusions: Nearly $50 \%$ of patients are being diagnosed with KD without meeting the full AHA criteria. Infants with cardiac involvement tend to present with incomplete AHA criteria. Irritability which is not among the AHA criteria should be considered as an important criterion for diagnosis in infants.

\section{G202 USEFULNESS OF SCREENING AND CONTACT TRACING IN} CHILDHOOD TUBERCULOSIS

Julia Clark, Catherine Barnaby, Anne Black, Elspeth Gould, Andrew Cant Paediatric Immunology and Infectious Diseases Unit (The Newcastle Upon Tyne Hospitals NHS Trust) Newcastle General Hospital, Newcastle, NE6 $4 B E$

De novo cases of tuberculosis (TB) make up $60 \%$ of our work load. Screening close contacts of adults with tuberculosis is also effective in identifying new cases. Referrals also come from the schools BCG service after positive Heaf tests. To date we have screened (i) all children in contact with TB, (ii) children in contact with Mantoux positive children without evidence of disease, (iii) those giving Heaf II-IV (no previous BCG) and (iv) Heaf III/IV (with BCG).

Aims: To find the proportion of children with old or active TB in each group.

Methods: Referrals from all groups were entered into a TB database. From this adults seen between 1990-31/3/99 and children between 1997-31/3/99 who were contacts of children either with TB or who were Mantoux positive were found, as was the number seen from schools between 1997-31/3/99 and the number between 1991 and 1996 estimated.

Results: (i) $4 \%(4 / 106)$ of adults and $6 \%(1 / 17)$ of children screened as contacts of child TB had TB. (ii) $\mathbf{0 . 3 \%}(4 / 1161)$ of adults and $0 \%(0 / 225)$ of children screened as contacts of children who were Mantoux positive without evidence of disease, had TB. (iii) Between 1991-9 1\%-1.3\% (8/612-795) of school Heaf II children had evidence of TB (mostly old) and from 1997-99 $\mathbf{0 . 8 \%}(2 / 255)$ had TB (old). (iv) Between 1991-99 3.2-4\% (7/171-219) school Heaf III/IV children had TB, mostly active, and 4.3\% (3/69) from 1997-9 had TB, mostly active. Importantly 5/8 of the school Heaf II positive children with TB had risk features e.g family history of TB, ethnicity, high incidence TB area.

Conclusion: In our population screening contacts of children with TB is highly worthwhile, whereas screening contacts of children who are Mantoux positive without disease is not. Screening those with Heaf III/IV responses at school is also rewarding; screening Heaf II responses at school is of borderline value yielding $1 \%$ mostly old TB, but appears more effective when only those from selected high risk populations are examined.

\section{G203 TEENAGE LIFESTYLE AND RISK OF MENINGOCOCCAL DISEASE}

Tully J, Booy R, Viner R Department of Epidemiology \& Public Health, Institute of Child Health, 30 Guilford Street, London WC1N 1EH

Background: The incidence of invasive meningococcal disease (MD) has increased in the UK. Adolescents are at particular risk and have a high mortality. Previous studies have included insufficient numbers of teenagers to clearly identify their risk factors. In particular, data on active smoking is scanty.

Aims: To elucidate social, biological and psychological risk factors for the development of MD in 15 to 19 year olds.

Methods: A prospective case-control study was performed across six regions of England. Subjects with a clinical diagnosis of MD were matched to GP-identified controls of the same age and sex. Structured questionnaires were completed asking about social behaviours in the two weeks prior to disease onset (cases) or interview (controls). Provisional results on 51 case control pairs are shown.

Results: $\mathrm{N}=51$ case control pairs. Mean age 17 years, $51 \%$ male, $96 \%$ white caucasian.

\begin{tabular}{llll}
\hline Activity & Cases & Controls & \\
\hline Active smoking & $37 \%$ & $31 \%$ & Non-sig \\
Presence of a partner & $43 \%$ & $33 \%$ & Non-sig \\
2 or more kissing contacts & $46 \%$ & $13 \%$ & $\mathrm{p}=0.006$ OR $=5.7$ \\
Partner who smokes & $48 \%$ & $11 \%$ & $\mathrm{p}=0.02$ OR $=7.9$ \\
\hline
\end{tabular}

Conclusions: Intimate contact and exposure to cigarette smoke increase the risk of invasive MD. These are behaviours that develop primarily during adolescence. The findings could inform public health campaigns that exploit the immediate risk from smoking to reduce smoking amongst adolescents.

\section{G204 HOSPITALIZATION FOR COMMUNITY-ACQUIRED} ROTAVIRUS-ASSOCIATED DIARRHOEA (RAD): A PROSPECTIVE LONGITUDINAL POPULATION-BASED STUDY

O'Hare, BAM, on behalf of Dr Ford-Jones, EL and the MetroToronto/Peel PRESI (Pediatric Rotavirus Epidemiology Study for Immunization) study group The Hospital for Sick Children, Toronto, Canada

Aims: To assess the need for rotavirus vaccine, contemporary populationbased information on the burden of rotavirus illness, including hospitalization rate, is required. A prospective multi-site cohort study was conducted to determine the age specific hospitalization rate for RAD during the seasonal outbreak in an Ontario, Canada pediatric population.

Methods: Children with an admission diagnosis of diarrhoea admitted to 18 hospitals serving 132 census tracts of the Greater Toronto/Peel region, between Nov. 1, 1997 to June 30, 1998. Prospective centralized testing of stools by ELISA and electron microscopy. Site records forwarded weekly to research nurses for identification of children with rotavirus and follow-up parent questionnaire administration.

Results: Of 224,160 children $<5 \mathrm{yr}$. in the region, the diarrhoea hospitalization rate was $4.8 / 1000(n=1086)$ during the seasonal epidemic. Based on testing of $65 \%$ of these admissions , the RAD hospitalization rates was 1.3 / 1000:the cumulative incidence to age 5yr. was 1/160. Hospitalization most commonly occurred in previously healthy children age 6 to $36 \mathrm{mo}$. The mean duration of rotavirus hospitalization based on hospital records and parental questioning respectively was $2.4+/-1.7$ and $3.6+/-1.6$ days. Hospitalization was significantly longer in children with an underlying medical condition. Diarrhoea occurred concurrently in $74 \%$ of household contacts $<3 y$ r., $38 \%$ age $3-18 y$ r., and $29 \%$ age $>18 y$ r.

Conclusions: Based on testing of $65 \%$ of children with diarrhoea, rotavirus resulted in hospitalization in a minimum of $1 / 160$ children by age 5 yr. during the 8 month seasonal outbreak. Had $100 \%$ of young children with diarrhoea been tested, the extrapolated cumulative incidence of rotavirus-associated diarrhoea by age $5 \mathrm{yr}$. may have been 1/106 during the 8 month period.

\section{G205 IMMUNISATION OF HOUSEHOLD CONTACTS OF WOMEN IDENTIFIED WITH HEPATITIS B BY ANTENATAL SCREENING}

V Clarke ${ }^{1}$, D Westmoreland ${ }^{2}$, Meirion R Evans ${ }^{3}{ }^{1}$ Department of Child Health, ${ }^{2}$ Public Health Laboratory, University Hospital of Wales, ${ }^{3}$ Public Health Directorate, Bro Taf Health Authority, , Cardiff 
Introduction: Universal antenatal screening for hepatitis B is now recommended in the United Kingdom, but there are concerns about adequacy of follow up. Children born to hepatitis B carrier women are at risk of both vertical and horizontal transmission of hepatitis $B$.

Aim: To assess the hepatitis B status and immunisation coverage of household contacts of women diagnosed to hepatitis B carriers on antenatal screening.

Methods: All hepatitis B carriers detected antenatally over a three year period were identified from laboratory records and sent a questionnaire to obtain details of household contacts and their hepatitis B immunisation status. Non-responders were contacted through the health visitor.

Results: Responses were received from 23/29 (79\%) women. Only 7/19 $(37 \%)$ current sexual partners and $5 / 11(45 \%)$ other adult household members had been fully immunised against hepatitis B. 4/12 unimmunised sexual partners had known hepatitis B status one was a hepatitis B carrier, one was naturally immune and two remained susceptible. Of 57 child contacts, 31 $(54 \%)$ were fully immunised, $13(23 \%)$ were partly immunised, $8(14 \%)$ were unimmunised and in five, immunisation status was unknown. Serum antibody tests had not been done in $28 / 57(49 \%)$ children and five $(9 \%)$ were reported to be hepatitis B carriers. Two children (4\%) had chronic liver disease.

Conclusions: Our study shows poor follow up and immunisation coverage in this high risk group. Unless proper follow up of hepatitis B carriers diagnosed by antenatal testing is achieved, the current United Kingdom policy of selective hepatitis $B$ immunisation is unlikely to succeed in eliminating hepatitis B.

Extra resources and health professionals with a defined responsibility are necessary to improve both immunisation coverage to this high risk group and awareness of the implications of hepatitis B carriage in these families. 